\title{
Complications of acute and chronic otitis media in the child - Review Article
}

\author{
Dra Mirta D Ambra* \\ WAMS Chairman, Especialist Minister Health Hospital Argerich UBA, Argentina
}

Received: 制July 08, 2018; Published: 阱July 16, 2018

*Corresponding author: Dra Mirta D Ambra, WAMS Chairman, Especialist Minister Health Hospital Argerich UBA, Argentina.

\begin{abstract}
Thanks to the discovery of antibiotics, the incidence of complications in the chronic and acute otitis media has significantly decreased, though they remain a potential mortality and morbidity risk. The reduction of complications can be conditional on some diagnostic delays, on account of lack of clinical suspicions, symptoms disguised by previous antibiotic treatments and wrong prognosis; therefore, the complications continue to be severe processes that endanger the patient's life. They are currently classified as extracranial and intracranial. The former is divided into extratemporal and intra temporal and represent a health problem for the pediatric population despite the extended use of antibiotics. The clinical, otomicroscopic and imaging assessment is reported as a diagnostic criterion predictive of suspicions and of confirmation. Facial paralysis, vertigo, vomiting, headache, pain irradiated to the mastoids, or to the temporoparietal region, and hyperthermia should be alerts to the physician about suppurative otitis complication.
\end{abstract}

\section{Epidemiology}

The incidence of complications of AOMs happened to be frequent and usually fatal, to rare, with low morbidity and mortality rate from the appearance of antibiotics. [1] reports that the complication rate in the OMA has declined significantly: 25 to $50 \%$ (it was preantibiotic), and after the years 80 (0.02 to $0.04 \%$ ), when all were treated with antimicrobials. After 1980, important indexes of complications are published again, especially in underdeveloped countries with extreme poverty. According to WHO data, AOM is described as a generally harmless disease, but very prevalent, since there are currently 120 million people in the world, mainly in developing countries, which suffer from it, and these figures include 7 million children.3 The increase in the incidence of extracranial and intracranial complications in the OMCC associated with loss hearing in pediatric patients. Current reports describe, according to a study of 22 years, 285 patients with AOM and OMC associated with otogenic complications, 1 and in another series, infectious complications of the temporal bone in patients under 20 years.

\section{Etiology}

The AOM is considered a frequent infectious process during the first 2 years of life. Most children have at least one episode before age school, and a third develop OMA, which can lead to serious complications

(mastoiditis, meningitis) and hyperacusis [2,3]. Factors that favor OMAR are relate to the male sex, the alterations of the immune response by part of the host, the short age (most frequently between 6 and 24 months) and start early onset of the first episode of otitis before the first 4-6 months of life. Anatomical characteristics of the Eustachian tube (TE) a wide, short, almost horizontal, flexible cartilaginous support and opening mechanism ineffective Immaturity of the external peristalsis muscle-, size of the nasopharynx, greater amount of regional lymphoid tissue that is hypertrophied by rhinopharyngeal infections repeated, which favors the tubal dysfunction and rise of secretions to the middle ear. Developmental defects, such as cleft palate or cleft lip-palate sequence, they affect TE functioning, ventilation and drainage of the middle ear cavity. An OM is produced with chronic effusion, and later formation of bags of retraction with invasion of the skin, chronic infection, cholesteatoma and possible extracranial and intracranial complications, in histological studies of Cholesteatoma of the middle ear, the presence of keratinized squamous epithelium. Among the genetic factors, it is discussed if what the genes transmit can be the 
propensity for disease report novel research on the genetic origin of pediatric cholesteatoma and its relation to mutations of the GJB2 and GJB6 gene. The most frequent pathogens in complications they are described as the same as those of the AOM: Streptococcus pneumoniae, Haemophilus infuenzae not capped and Moraxella catarrhalis [4]. Other germs involved in the complicated OMA, such as Streptococcus pyogenes, is currently reported to rarely causes mastoiditis. Staphylococcus aureus has a role less significant, and others such as E. coli, Pseudomonas aeruginosa and anaerobes are they consider less frequent. [3] describe the Streptococcus pneumoniae as the main cause of complicated OMA in patient's pediatric patients, and their resistance to penicillin's is on the rise in the world. Among the factors that influence the complications of AOM are the type and virulence of the infecting microorganism (Streptococcus pneumoniae and different serotypes), decreases host resistance, susceptibility to medications anti-bacterial available for treatment or insufficient dose, as well as an inadequate administration time and medication selection Meningitis, independently from its origin, can cause labyrinthitis through natural pathways, especially the CAI, who will study bilaterally and will cause deafness in pre-lingual ages. It is diagnosed by CAT that evaluates ear abnormalities. Internal (fistula), MRI to evaluate soft tissues of the CNS, and lumbar puncture (LP) for cytochemical study and culture of CSF, where it is appreciated: pleocytosis, abundant polymorphonuclear cells, decreased glucorquine and moderate level increase protein Staining should be performed by Gram technique, microscopic study and culture with antibiogram to detect causal germ and adequate treatment. Intravenous treatment is recommended cephalosporins of third generation, of choice ceftriazona, tímpanocentesis moiringotomía for culture and drainage.

\section{Conclusion}

The study by residents, general practitioners and novice specialists of Otorhinolaryngology and Pediatrics, of the OMA and chronicles and their complications, is fundamental for early diagnosis and early intervention, which contributes to avoid or reduce the complications of $\mathrm{OM}$, with great impact on the quality of life of children

\section{References}

1. Zalzal G (2007) Complications of acute otitis media. In: Sih T, ChinskiA, Eavey R, Godinho R (Eds.), Pediatric Otolaryngology Manual of the IAPO. Brazil Life \& Consciência pp. 280-282.

2. Quintero JL (2011) Chronic otitis media. In: De la Torre E, Pelayo EJ, editors. Pediatrics. V6. Havana: Editorial Medical Sciences pp. 2644-2654.

3. Bakhos D, Trijolet JP, Morinieri S, Pondaven S, Al Zahrani M (2011) Conservative management of acute mastoiditis in children. Arch Otolaryngol Head Neck Surg 137(4): 346-350

4. De la Flor J (2009) Upper respiratory tract infections II. Otitis media: etiology, clinical, diagnosis, complication and treatment; Recurrent otitis media and otitis media chronicle; external otitis. In: Prandi F, Del Pozo J, Hidalgo I, De la Flor J, editores, et al. (Eds.), Comprehensive Pediatrics V XIII. Madrid: Ergon pp. 333-355.

5. Garcés Sánchez M, Diez Domingo J, Álvarez de Labiada T, Planelles V, Graullera M, et al. (2004) Epidemiology and impact of acute otitis media in the community. Valenciana An Pediatric Barc 60(2): 125-132.

6. Dubey SP, Larawin V (2007) Complications of chronic suppurative otitis media. Laryngoscope 117(2): 264-267.

7. Wu JF, Jin Z, Yang JM, Liu YH, Duan ML (2012) Extracranialand intracranial of otitis media: 22 year clinical experience and analysis. Otolaryngology Act 132(3): 261-265.

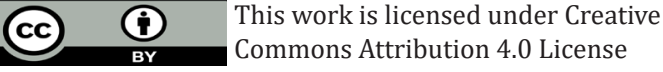

To Submit Your Article Click Here:

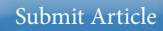

DOI: $10.32474 /$ SJO.2018.01.000104

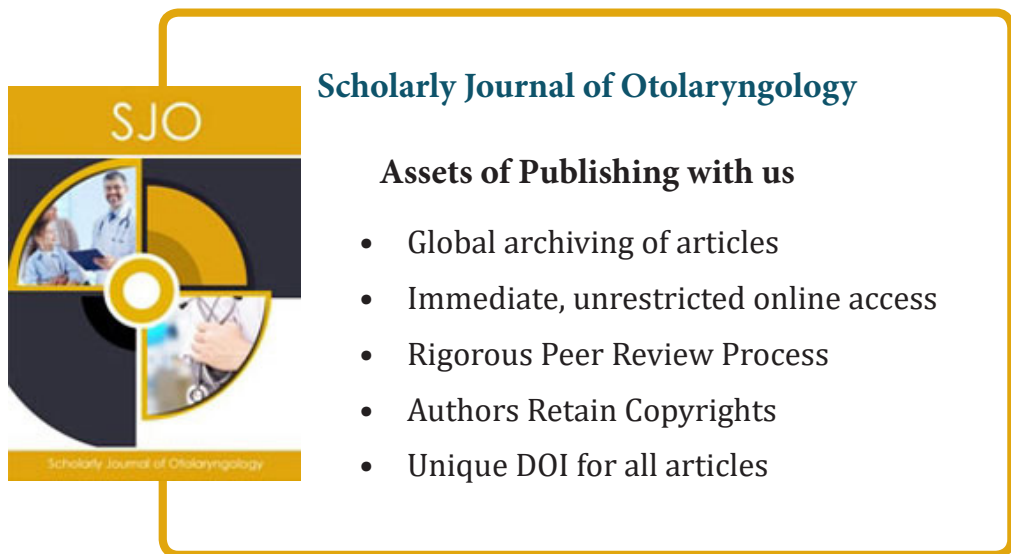

